\title{
Orbital Recurrence following Aggressive Laser Treatment for Recurrent Retinoblastoma
}

\author{
Bradley H. Jacobsen ${ }^{a}$ Jesse L. Berry b, c Rima Jubranc Jonathan W. Kimb, c \\ a University of California, Irvine School of Medicine, Irvine, Calif., and ${ }^{b}$ USC Eye Institute, Keck School of Medicine, \\ University of Southern California, and ' Children's Hospital Los Angeles, Los Angeles, Calif., USA
}

\section{Key Words}

Retinoblastoma - Tumor recurrence · Focal therapy · Diode laser · Argon laser · Oncology

\section{Abstract}

Purpose: To report a case of a 29-month-old male with familial, bilateral retinoblastoma with orbital recurrence following aggressive laser treatment. Methods: This is a retrospective case report of a single patient. Results: A 3-week-old male was diagnosed with familial, bilateral retinoblastoma (group D OD, group A OS), and treated with 6 cycles of systemic chemotherapy and laser treatment. The patient exhibited full regression of tumors in both eyes. Two years after diagnosis, a small tumor recurrence within the macular laser scar of the right eye was discovered. The tumor recurrence was treated with argon and diode laser over 6 months. The tumor recurrence completely resolved following the laser treatments. Ten weeks after the last laser treatment, MRI revealed a large intraconal orbital mass, contiguous with the sclera of the right eye. Orbital biopsy and systemic work-up confirmed the diagnosis of retinoblastoma with no other metastatic foci. B-scan ultrasonography showed thinning of the sclera at the site of recurrence. Conclusion: This is an un- usual orbital recurrence after successful treatment of intraocular disease with aggressive laser therapy. Clinicians should be aware of the rare possibility of orbital recurrence following chemoreduction with local consolidation even when fundus examinations are normal.

(c) 2015 S. Karger AG, Basel

\section{Introduction}

Retinoblastoma is the most common intraocular malignancy in children and represents roughly $4 \%$ of pediatric malignancies [1]. In the early 1900 s, the prognosis for children with retinoblastoma was a mere $5 \%$, but this has dramatically improved to $95 \%$ in developed countries [2].

Orbital recurrence of retinoblastoma following successful treatment of intraocular disease is extremely rare. Herein, we present a patient who was successfully treated with chemotherapy and local consolidation followed by aggressive laser therapy for a macular recurrence and who subsequently developed an unusual type of orbital recurrence.

\section{KARGER}

E-Mail karger@karger.com www.karger.com/oop
(C) 2015 S. Karger AG, Base

2296-4681/15/0022-0076\$39.50/0
Bradley Jacobsen, BS

University of California School of Medicine

5050 E Garford \#118

Long Beach, CA 90815 (USA)

E-Mail bjacobse@uci.edu 


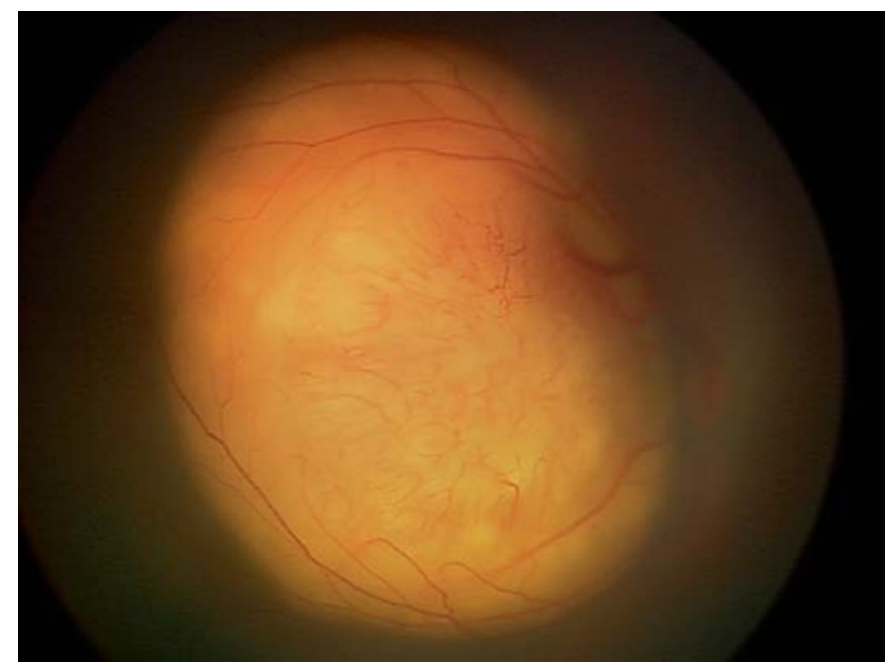

Fig. 1. Macular group $C$ retinoblastoma of the right eye.

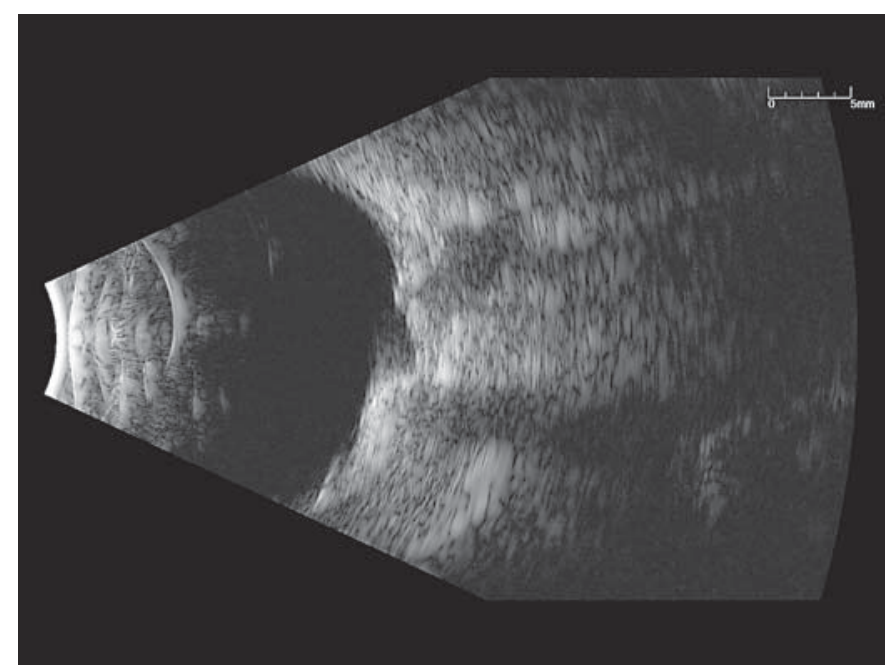

Fig. 3. Ocular ultrasound performed after laser treatment for recurrence, which reveals scleral thinning.

\section{Case Presentation}

A 3-week-old male underwent an examination under anesthesia (EUA) at the Children's Hospital Los Angeles with a positive family history of bilateral retinoblastoma in his father and older brother. Past medical history was otherwise completely negative, and review of systems was also unremarkable. He was born fullterm without any complications during pregnancy or delivery. No prenatal or in utero genetic testing for mutations in the retinoblastoma gene had been performed prior to the presentation.

Initial EUA revealed group $C$ unilateral retinoblastoma in the right eye with a macular tumor and surrounding subretinal fluid



Fig. 2. Recurrent tumor in the atrophic macular scar of the right eye.

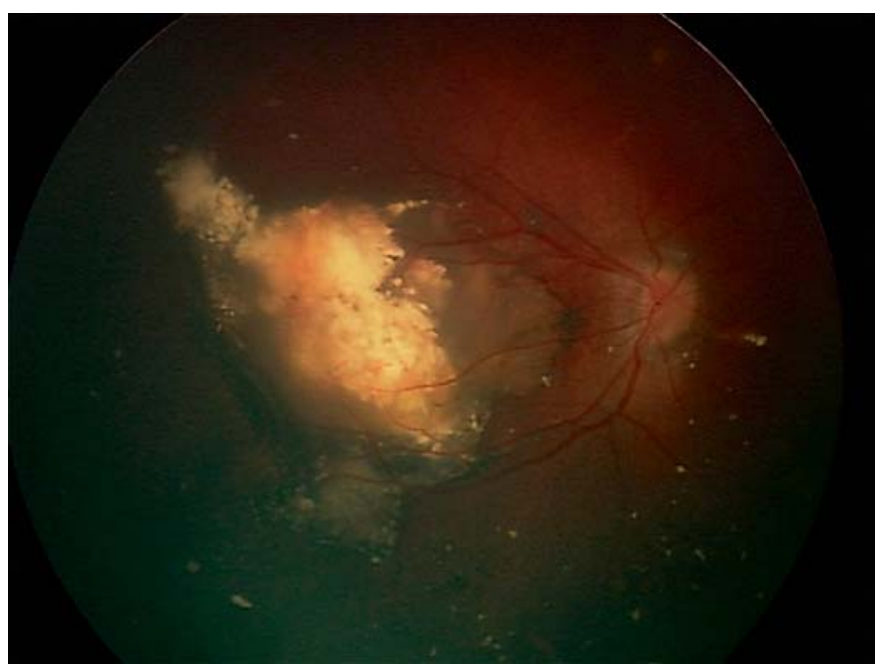

Fig. 4. Completely regressed macular tumor after laser treatment (right eye).

(fig. 1). The left eye was normal without evidence of retinoblastoma at initial evaluation. MRI of the brain showed the macular tumor but no extraocular extension. The patient completed 6 cycles of systemic chemotherapy and laser treatment with excellent regression of the tumor. At 9 months of age, the patient was discovered to have a small retinoblastoma tumor in the left eye (group A) at the ora serrata. The new tumor focus was successfully treated with red diode laser $(230 \mathrm{~mW})$.

Two years after the initial diagnosis, a recurrent tumor was noted in the atrophic macular laser scar in the right eye (fig. 2). Ocular ultrasound performed at this time demonstrated scleral thinning (fig. 3). Intra-arterial chemotherapy was attempted, but the oph- 




Fig. 5. MRI scan of right eye demonstrating large intraconal mass, located directly behind the sclera of the right eye.

thalmic artery could not be successfully cannulated. The tumor recurrence was then treated with laser therapy over a total of 10 sessions over the next 6 months. Diode and argon laser units with power levels ranging between $400-900 \mathrm{~mW}$ were used. The nodule of active tumor resolved completely (fig. 4).

An EUA 10 weeks after the last laser treatment showed no evidence of intraocular disease. However, a routine screening MRI scan performed at that time showed a large intraconal mass located directly posterior to the globe and adjacent to the sclera of the right eye (fig. 5). An orbital biopsy was performed through a conjunctival approach, which confirmed the diagnosis of orbital retinoblastoma. Metastatic workup was negative for bone, bone marrow, or central nervous system disease. Follow-up MRI scan after the completion of systemic, globe-conserving treatment showed complete resolution of the orbital mass. Fundus examinations continue to show no evidence of active intraocular disease.

\section{Discussion}

Advances in systemic and local therapies to treat retinoblastoma have decreased the need for enucleation. However, these therapies also have the potential for complications [3]. Laser treatment for retinoblastoma is widely accepted among ocular oncologists, either as a standalone therapy (usually for small tumors) or in combination with chemoreduction. However, treatment parameters (number of sessions, power of laser, etc.) for various clinical settings have not been standardized [4]. Tawansy et al. [3] described numerous cases of patients treated with systemic chemotherapy and focal treatment (with laser) who then developed vitreoretinal complications. These complications included vitreous traction band, subretinal fibrosis, preretinal fibrosis, retinal detachment, and pseudo-vitreous seeding. Other intraocular complications following laser treatment for retinoblastoma include vitreous and retinal hemorrhage, retinal contraction, and rhegmatogenous retinal detachment.

Another source of concern following aggressive laser treatment is relapse of tumor. It has been suggested that high-energy focal treatment can disrupt the internal limiting membrane causing dispersion of malignant cells from the tumor [5]. The risk of extraocular extension of retinoblastoma following the violation of the sclera has been well described when performing intraocular surgical procedures [6], although never previously reported following laser treatment. For example, Karcioglu et al. [7] found evidence of tumor seeding in the needle tracks following aspiration of intraocular retinoblastoma tumors. In our case, we postulate that repeated, aggressive laser treatment caused a focal thinning of the sclera within the atrophic scar, allowing a portal of entry for active tumor cells to seed the orbit. Another possibility is that the orbital recurrence was related to metastatic disease caused by hematogenous dissemination to the orbit [8]. We consider this mechanism less likely given the original diagnosis of group $\mathrm{C}$ retinoblastoma and the location of the single extraocular lesion directly posterior to the sclera with no other evidence of metastatic disease.

Focal therapy for retinoblastoma has many advantages including salvage of the globe with negligible systemic or regional complications. However, the short- and longterm implications of these treatments after chemoreduction are still being evaluated. Our case report highlights a potential consequence of aggressive focal therapy and serves as an example of the possibility of orbital tumor recurrence in retinoblastoma patients even when intraocular examinations show no clinical evidence of tumor activity. 


\section{Statement of Ethics}

This study was conducted in compliance with the rules and regulations of the Health Insurance Portability and Accountability Act as well as in adherence with the Declaration of Helsinki and all other relevant federal and state laws.

\section{Disclosure Statement}

The authors declare that they have no conflicts of interests or financial disclosures.

\section{References}

1 Castillo BV Jr, Kaufman L: Pediatric tumors of the eye and orbit. Pediatr Clin North Am 2003;50:149-172.

2 Shields CL, Honavar S, Shields JA, Demirci H, Meadows AT: Vitrectomy in eyes with unsuspected retinoblastoma. Ophthalmology 2000; 107:2250-2255.

3 Tawansy KA, Samuel MA, Shammas M, Muprhree AL: Vitreoretinal complications of retinoblastoma treatment. Retina 2006;26:S47S52.
4 Hamel P, Heon E, Gallie BL, Budning AS: Focal therapy in the management of retinoblastoma: when to start and when to stop. J AAPOS 2000;4:334-337.

5 Gombos DS, Cauchi PA, Hungerford JL, Addison P, Coen PG, Kingston JE: Vitreous relapse following primary chemotherapy for retinoblastoma: is adjuvant diode laser a risk factor? Br J Ophthalmol 2006;90:1168-1172.
6 Stevenson KE, Hungerford J, Garner A: Local extraocular extension of retinoblastoma following intraocular surgery. Br J Ophthalmol 1989;73:739-742.

7 Karcioglu ZA, Gordon RA, Karcioglu GL: Tumor seeding in ocular fine needle aspiration biopsy. Ophthalmology 1985;92:1763-1767.

8 Kim JW, Kathpalia V, Dunkel IJ, Wong RK, Riedel E, Abramson DH: Orbital recurrence of retinoblastoma following enucleation. $\mathrm{Br} \mathrm{J}$ Ophthalmol 2009;93:463-467. 